\title{
Utilização de compostos orgânicos como substratos na produção de mu- das de hortaliças
}

\author{
Marco Antonio de A Leal; José Guilherme M Guerra; Ricardo TG Peixoto; Dejair L de Almeida \\ Embrapa Agrobiologia, BR 465, km 7, 23851-970 Seropédica-RJ; mleal@cnpab.embrapa.br
}

\begin{abstract}
RESUMO
Visando determinar a viabilidade da utilização de compostos orgânicos obtidos com palhada de Crotalaria juncea L. e capim Napier (Pennisetum purpureum Schum.) como substratos na produção de mudas de hortaliças, realizaram-se experimentos com alface (folhosa), beterraba (raiz) e tomate (hortaliça de fruto). Estudou-se a eficiência de compostos produzidos a partir dos materiais: 100\% de Crotalária Júncea; 66\% de Crotalária Júncea + 33\% de Napier; 33\% de Crotalária Júncea + 66\% de Napier; 100\% de Napier; 33\% de Crotalária Júncea $+66 \%$ de Napier, inoculado com $5 \%$ da massa com esterco bovino; 33\% de Crotalária Júncea + 66\% de Napier, inoculado com 100 L de Agrobio diluído a 5\%; 100\% de Napier, inoculado com 100 litros de Agrobio diluído a 5\%. Como controle utilizou-se o substrato comercial Plantmax HT®. Avaliou-se altura da parte aérea, número de folhas, produção de massa fresca na parte aérea e produção de massa seca na parte aérea. O composto produzido com a mistura de $66 \%$ de Crotalária Júncea e 33\% de Napier mostrou-se superior aos demais tratamentos para produção de mudas de alface, beterraba e tomate.
\end{abstract}

Palavras-chave: Crotalaria juncea L., Pennisetum purpureum Schum., compostagem, mudas, produção orgânica.

\begin{abstract}
Utilization of organic compost as substrate for vegetable seedling production

The viability of the organic compost utilization as a substrate for vegetable seedlings production, obtained from Crotalaria juncea L. and Napier grass (Pennisetum purpureum Schum.), was determined. The research was developed with lettuce (leaf vegetable), beetroot (root vegetable) and tomato (fruit vegetable). The organic composts were produced from: 100\% Crotalaria Juncea; 66\% Crotalaria Juncea $+33 \%$ Napier; 33\% Crotalaria Juncea $+66 \%$ Napier; 100\% Napier; 33\% Crotalaria Juncea + 66\% Napier, inoculated with $5 \%$ of the mass with cattle manure; $33 \%$ Crotalaria Juncea + 66\% Napier, inoculated with 100 L of 5\% diluted Agrobio; $100 \%$ Napier, inoculated with $100 \mathrm{~L}$ of $5 \%$ diluted Agrobio. We evaluated the height, leaf number, aerial green weight and aerial dry weight. A commercial substrate Plantmax HT® was used as control. The compost produced from the mixture of $66 \%$ Crotalaria Juncea and 33\% Napier presented the best results, being adequate for the production of lettuce, beetroot and tomato seedlings.
\end{abstract}

Keywords: Crotalaria juncea L., Pennisetum purpureum Schum., composting, seedlings, organic production.

\section{(Recebido para publicação em 17 de maio de 2006; aceito em 11 de setembro de 2007)}

ث́ crescente a demanda por substratos, utilizados principalmente na produção de plantas ornamentais, hortaliças em recipientes e mudas (Abreu et al., 2002). Grande parte dos substratos são produzidos utilizando-se turfa como componente principal, mas são crescentes os esforços visando a substituição deste material, devido a questões de proteção ambiental (Baumgarten, 2002). Os compostos orgânicos podem atender plenamente esta demanda, principalmente em sistemas orgânicos de produção, que impedem o uso de fertilizantes sintéticos de elevada solubilidade.

Devido ao limitado volume para o crescimento das raízes, os substratos devem ser capazes de proporcionar fornecimento constante de água, oxigênio e nutrientes para as plantas (Fermino, 2002), garantido assim ambientes estáveis para o desenvolvimento das plantas (Carlile, 1997).

Compostos orgânicos podem fornecer os nutrientes necessários ao cresci- mento para várias culturas. Embora os compostos orgânicos possam conter quantidades significativas de $\mathrm{N}$, a maior parte se encontra na forma orgânica e não está plenamente disponível para as plantas (WRAP, 2004). Quando a demanda total de $\mathrm{N}$ pela planta é baixa e distribuída por um longo período de tempo, a utilização de compostos orgânicos como substratos pode fornecer todo o $\mathrm{N}$ necessário. Caso contrário, também devem ser utilizados fertilizantes com rápida disponibilização de N. O mesmo vale para os outros nutrientes (Hadas \& Portnoy, 1997). Os compostos orgânicos geralmente possuem CTC mais elevada do que a turfa e possuem efeito corretivo do pH (WRAP, 2004).

Os compostos orgânicos devem possuir boas propriedades físicas para serem utilizados como substrato. Uma importante característica é a alta capacidade de reter a umidade e drenar o excesso de água (Corti \& Crippa, 1998). O substrato também deve promover de forma adequada o fornecimento de oxigênio e a eliminação do $\mathrm{CO}_{2}$ (WRAP, 2004). Outra característica física importante para a utilização de compostos orgânicos como substrato, é estes possuírem reduzido grau de contração ou expansão.

Os compostos orgânicos possuem propriedades biológicas adequadas para seu uso como substratos. Existe na literatura a evidência de que os compostos podem estimular a proliferação de antagonistas a organismos fitopatogênicos, ajudando a controlar algumas doenças do sistema radicular (De Brito \& Gagne, 1995; Mandelbaum \& Hadar, 1997; Lievens, 2001).

Este trabalho visou avaliar a viabilidade do uso de compostos orgânicos obtidos com palhada de Crotalaria juncea L. e capim Napier (Pennisetum purpureum Schum.) como substratos inteiramente orgânicos para a produção de mudas de hortaliças. 


\section{MATERIAL E MÉTODOS}

A produção dos compostos utilizados nos tratamentos iniciou-se em março de 2004 e terminou após três meses de compostagem. As pilhas foram montadas com dimensões de 2,0 x 2,0 x 1,2 $\mathrm{m}\left(4,8 \mathrm{~m}^{3}\right)$, a céu aberto, sob lona plástica, em quatro camadas contendo todos os materiais.

As matérias-prima utilizadas foram 1) parte aérea de Crotalaria juncea L. com três meses de idade, cortada e fragmentada em picadeira uma semana antes da montagem das pilhas; 2) capim Napier (Pennisetum purpureum Schum.) cortado e fragmentado em picadeira duas semanas antes da montagem das pilhas; 3) esterco bovino curtido; e 4) biofertilizante líquido Agrobio, produzido à base de esterco, melaço, torta de mamona e micronutrientes. A proporção de cada matéria prima foi calculada com base no seu teor de matéria seca.

A irrigação das pilhas foi realizada sempre que amostragens semanais revelavam que a umidade se encontrava abaixo de $50 \%$. O revolvimento das pilhas foi realizado semanalmente no primeiro mês e quinzenalmente no segundo e terceiro mês.

Foram avaliados os compostos produzidos a partir dos materiais a) 100C: 100\% de Crotalária Júncea; b) 66C33N: 66\% de Crotalária Júncea + 33\% de Napier; c) 33C66N: 33\% de Crotalária Júncea + 66\% de Napier; d) 100N: $100 \%$ de Napier; e) 33C66N+E: 33\% de Crotalária Júncea + 66\% de Napier, inoculado com mais 5\% da massa com esterco bovino; f) $33 \mathrm{C} 66 \mathrm{~N}+\mathrm{A}$ : 33\% de Crotalária Júncea + 66\% de Napier, inoculado com 100 litros de Agrobio diluído a 5\%; g) $100 \mathrm{~N}+\mathrm{A}$ : $100 \%$ de Napier, inoculado com 100 litros de Agrobio diluído a 5\%. Além dos compostos, foi incluído como tratamento o substrato comercial Plantmax HT® como controle. A caracterização destes materiais está apresentada na Tabela 1. A análise de $\mathrm{pH}$ foi realizada segundo o método descrito por Tedesco (1995), em solução de água destilada. A condutividade elétrica foi medida no mesmo extrato aquoso obtido para a medição do pH (5:1, v/v).

Tabela 1. Valores de pH, CE e teores de N, Ca, Mg, P e K obtidos dos materiais utilizados como substratos ( $\mathrm{Ph}, \mathrm{CE}$ and content of $\mathrm{N}, \mathrm{Ca}, \mathrm{Mg}, \mathrm{P}$ and $\mathrm{K}$, obtained from the utilized substrata). Seropédica, Embrapa Agrobiologia, 2004.

\begin{tabular}{|c|c|c|c|c|c|c|c|}
\hline & \multirow{2}{*}{$\mathrm{pH}$} & \multirow{2}{*}{$\begin{array}{c}\text { CE } \\
\left(\mathrm{dS} \mathrm{m}^{-1}\right)\end{array}$} & $\mathbf{N}$ & $\mathrm{Ca}$ & $\mathbf{M g}$ & $\mathbf{P}$ & $\mathbf{K}$ \\
\hline & & & \multicolumn{5}{|c|}{$\left(\mathrm{g} \mathrm{kg}^{-1)}\right.$} \\
\hline $100 \mathrm{C}$ & 7,1 & 2,8 & 40,0 & 9,9 & 5,9 & 9,5 & 4,5 \\
\hline $66 \mathrm{C} 33 \mathrm{~N}$ & 7,5 & 2,0 & 36,9 & 7,7 & 4,1 & 8,5 & 3,6 \\
\hline $33 \mathrm{C} 66 \mathrm{~N}$ & 8,1 & 1,9 & 30,6 & 7,5 & 4,0 & 10,3 & 3,6 \\
\hline $100 \mathrm{~N}$ & 8,4 & 1,6 & 9,6 & 4,2 & 2,3 & 9,5 & 2,3 \\
\hline $33 \mathrm{C} 66 \mathrm{~N}+\mathrm{E}$ & 8,2 & 2,0 & 29,1 & 3,7 & 1,6 & 2,6 & 3,3 \\
\hline $33 \mathrm{C} 66 \mathrm{~N}+\mathrm{A}$ & 8,1 & 1,9 & 29,6 & 8,1 & 5,2 & 12,5 & 6,1 \\
\hline $100 N+A$ & 8,7 & 1,3 & 10,2 & 4,2 & 1,5 & 6,2 & 1,7 \\
\hline Controle & 6,5 & 1,8 & 5,6 & 18,1 & 17,6 & 2,2 & 4,1 \\
\hline
\end{tabular}

Tabela 2. Valores de altura, número de folhas, produção de massa fresca e seca da parte aérea, obtidos para as mudas de alface aos 33 dias após a semeadura (plant height, number of leaves, production of fresh and dry mass of the aerial part obtained from lettuce plantlets, 33 days after sowing date). Seropédica, Embrapa Agrobiologia, 2004.

\begin{tabular}{|c|c|c|c|c|}
\hline & Altura (cm) & $N^{\circ}$ de folhas & $\begin{array}{l}\text { Massa fresca } \\
\text { da parte aérea }\end{array}$ & $\begin{array}{c}\text { Massa seca da } \\
\text { parte aérea }\end{array}$ \\
\hline & & & \multicolumn{2}{|c|}{$\left(\right.$ mg planta $\left.^{-1}\right)$} \\
\hline $100 C^{2}$ & $2,4 \mathrm{C}^{1}$ & $6,7 \mathrm{~B}$ & $186,7 \mathrm{~B}$ & $15,3 \mathrm{~B}$ \\
\hline $66 \mathrm{C} 33 \mathrm{~N}$ & $5,4 \mathrm{~A}$ & $8,5 \mathrm{~A}$ & $603,3 \mathrm{~A}$ & $53,2 \mathrm{~A}$ \\
\hline $33 \mathrm{C} 66 \mathrm{~N}$ & $3,1 \mathrm{C}$ & $7,5 \mathrm{~B}$ & 306,6 B & $26,0 \mathrm{~B}$ \\
\hline $100 N$ & $0,8 \mathrm{D}$ & $3,0 \mathrm{C}$ & $16,7 \mathrm{C}$ & $1,9 \mathrm{C}$ \\
\hline $33 \mathrm{C} 66 \mathrm{~N}+\mathrm{E}$ & $4,4 \mathrm{~B}$ & $8,1 \mathrm{AB}$ & $550,0 \mathrm{~A}$ & $45,9 \mathrm{~A}$ \\
\hline $33 \mathrm{C} 66 \mathrm{~N}+\mathrm{A}$ & $4,9 \mathrm{AB}$ & $8,3 \mathrm{~A}$ & $633,3 \mathrm{~A}$ & $52,1 \mathrm{~A}$ \\
\hline $100 N+A$ & $0,9 \mathrm{D}$ & $2,9 \mathrm{C}$ & $20,0 \mathrm{C}$ & $1,5 \mathrm{C}$ \\
\hline Controle & $2,8 \mathrm{C}$ & $7,1 \mathrm{~B}$ & $230,0 \mathrm{~B}$ & $22,7 \mathrm{~B}$ \\
\hline $\mathrm{CV} \%$ & 10,3 & 3,8 & 16,4 & 18,8 \\
\hline
\end{tabular}

${ }^{1}$ Médias na mesma coluna seguidas de diferentes letras são diferentes ( $\left.\mathrm{p} £ 0,05\right)$ pelo teste Tukey; ²100C: 100\% de Crotalária Júncea; 66C33N: 66\% de Crotalária Júncea + 33\% de Napier; 33C66N: 33\% de Crotalária Júncea + 66\% de Napier; 100N: 100\% de Napier; 33C66N+E: 33\% de Crotalária Júncea + 66\% de Napier, inoculado com mais 5\% da massa com esterco bovino; 33C66N+A: 33\% de Crotalária Júncea + 66\% de Napier, inoculado com 100 litros de Agrobio diluído a 5\%; 100N+A: 100\% de Napier, inoculado com 100 litros de Agrobio diluído a $5 \%$ (mean values in the column, followed by the same letter did not differ from each other in the Tukey test; ${ }^{2} 100 C$ : 100\% Crotalária Júncea; 66C33N: 66\% Crotalária Júncea + 33\% Napier; 33C66N: 33\% Crotalária Júncea + 66\% Napier; 100N: 100\% Napier; 33C66N+E: $33 \%$ Crotalária Júncea $+66 \%$ Napier, innoculated with 5\% of the mass of cattle manure; 33C66N+A: 33\% Crotalária Júncea + 66\% Napier, innoculated with 100L of 5\% diluted Agrobio; 100N+A: 100\% Napier, inoculado com 100 L of 5\% diluted Agrobio).

As análises dos teores de N, Ca, Mg, Ke P foram realizadas utilizando o procedimento operacional descrito por Silva (1999).

Utilizou-se as espécies: alface (folhosa) cultivar "Regina”, beterraba (raiz) cultivar “Top Early Wonder” e tomate (hortaliça de fruto) cultivar "Santa Clara”. Foi realizado um experimento para cada espécie. Os experimentos foram instalados em delineamento inteiramente casualizado, com três repetições.

Os experimentos foram realizados em casa de vegetação da PESAGRO RIO, situada em Seropédica-RJ, a 26 m de altitude e coordenadas $22^{\circ} 45^{\prime} \mathrm{S}$ (latitude) e $43^{\circ} 40^{\prime} \mathrm{W}$ (longitude). As mudas de tomate e de beterraba foram produzidas em bandejas de poliestireno expandido com 128 células e as mudas de alface em bandejas com 200 células. Para cada tratamento foi utilizada a metade de uma bandeja. Visando reduzir influências locais, as bandejas foram trocadas de lugar duas vezes por semana.

A semeadura ocorreu em 24/06/04 e as avaliações ocorreram 33 dias após a 
Tabela 3. Valores de altura, número de folhas, produção de massa fresca e seca da parte aérea, obtidos em mudas de beterraba aos 33 dias após a semeadura (plant height, number of leaves, fresh and dry mass of aerial part, obtained from beetroot plantlets, 33 days after sowing date). Seropédica, Embrapa Agrobiologia, 2004.

\begin{tabular}{|c|c|c|c|c|}
\hline & Altura $(\mathrm{cm})$ & $N^{\circ}$ de folhas & $\begin{array}{l}\text { Massa fresca } \\
\text { da parte aérea }\end{array}$ & $\begin{array}{c}\text { Massa seca da } \\
\text { parte aérea }\end{array}$ \\
\hline & & & \multicolumn{2}{|c|}{ (mg planta-1) } \\
\hline $100 C^{2}$ & $4,4 \mathrm{D}$ & $3,7 \mathrm{~B}$ & $210,0 D$ & $11,6 \mathrm{CD}$ \\
\hline $66 \mathrm{C} 33 \mathrm{~N}$ & $10,3 \mathrm{~A}$ & $4,6 \mathrm{~A}$ & $870,0 \mathrm{~A}$ & $54,7 \mathrm{~A}$ \\
\hline $33 \mathrm{C} 66 \mathrm{~N}$ & $9,1 \mathrm{AB}$ & $4,1 \mathrm{~B}$ & 643,3 B & $37,0 \mathrm{~B}$ \\
\hline $100 \mathrm{~N}$ & $2,4 \mathrm{E}$ & $2,0 \mathrm{C}$ & $30,0 \mathrm{E}$ & $3,3 \mathrm{D}$ \\
\hline $33 \mathrm{C} 66 \mathrm{~N}+\mathrm{E}$ & $6,8 \mathrm{C}$ & $4,0 \mathrm{~B}$ & $373,3 \mathrm{C}$ & $21,3 \mathrm{C}$ \\
\hline $33 \mathrm{C} 66 \mathrm{~N}+\mathrm{A}$ & $7,0 \mathrm{C}$ & $3,9 \mathrm{~B}$ & $376,7 \mathrm{C}$ & $21,6 \mathrm{C}$ \\
\hline $100 N+A$ & $2,5 \mathrm{E}$ & $2,0 \mathrm{C}$ & $30,0 \mathrm{E}$ & $2,8 \mathrm{D}$ \\
\hline Controle & $9,0 \mathrm{~B}$ & $4,1 \mathrm{~B}$ & 646,7 B & $46,3 \mathrm{AB}$ \\
\hline CV\% & 7,1 & 4,4 & 14,3 & 15,3 \\
\hline
\end{tabular}

${ }^{1}$ Médias na mesma coluna seguidas de diferentes letras são diferentes $(\mathrm{p} £ 0,05)$ pelo teste Tukey; ${ }^{2}$ 100C: 100\% de Crotalária Júncea; 66C33N: 66\% de Crotalária Júncea + 33\% de Napier; 33C66N: 33\% de Crotalária Júncea + 66\% de Napier; 100N: 100\% de Napier; 33C66N+E: $33 \%$ de Crotalária Júncea $+66 \%$ de Napier, inoculado com mais 5\% da massa com esterco bovino; 33C66N+A: 33\% de Crotalária Júncea + 66\% de Napier, inoculado com 100 litros de Agrobio diluído a 5\%; 100N+A: 100\% de Napier, inoculado com 100 litros de Agrobio diluído a $5 \%$ (mean values in the column, followed by the same letter did not differ from each other in the Tukey test (p£0,05); ${ }^{2} 100 C$ : 100\% Crotalária Júncea; 66C33N: 66\% Crotalária Júncea + 33\% Napier; 33C66N: 33\% Crotalária Júncea + 66\% Napier; 100N: 100\% Napier; 33C66N+E: $33 \%$ Crotalária Júncea $+66 \%$ Napier, innoculated with $5 \%$ of the mass of cattle manure; 33C66N+A: 33\% Crotalária Júncea + 66\% Napier, innoculated with 100L of 5\% diluted Agrobio; $100 \mathrm{~N}+\mathrm{A}$ : 100\% Napier, inoculado com $100 \mathrm{~L}$ of 5\% diluted Agrobio).

Tabela 4. Valores de altura, número de folhas, produção de massa fresca e seca da parte aérea, obtidos para as mudas de tomate aos 33 dias após a semeadura (plant height, number of leaves, yield of fresh and dry mass of the aerial part, obtained 33 days after sowing date). Seropédica, Embrapa Agrobiologia, 2004.

\begin{tabular}{lcccc}
\hline & Altura $(\mathbf{c m})$ & $\mathbf{N}^{\circ}$ de folhas & $\begin{array}{c}\text { Massa fresca } \\
\text { da parte aérea }\end{array}$ & $\begin{array}{c}\text { Massa seca da } \\
\text { parte aérea }\end{array}$ \\
\cline { 4 - 5 } & & $4,9 \mathrm{~B}$ & $363,3 \mathrm{D}$ & $33,7 \mathrm{C}$ \\
\hline $100 \mathrm{C}^{2}$ & $5,0 \mathrm{D}$ & $5,7 \mathrm{~A}$ & $1020,0 \mathrm{~A}$ & $119,2 \mathrm{~A}$ \\
$66 \mathrm{C} 33 \mathrm{~N}$ & $10,2 \mathrm{~A}$ & $5,0 \mathrm{~B}$ & $553,3 \mathrm{C}$ & $62,3 \mathrm{~B}$ \\
$33 \mathrm{C} 66 \mathrm{~N}$ & $7,3 \mathrm{C}$ & $2,1 \mathrm{C}$ & $20,0 \mathrm{E}$ & $2,3 \mathrm{D}$ \\
$100 \mathrm{~N}$ & $2,3 \mathrm{E}$ & $5,0 \mathrm{~B}$ & $540,0 \mathrm{C}$ & $62,5 \mathrm{~B}$ \\
$33 \mathrm{C} 66 \mathrm{~N}+\mathrm{E}$ & $7,0 \mathrm{C}$ & $5,0 \mathrm{~B}$ & $476,7 \mathrm{C}$ & $53,8 \mathrm{~B}$ \\
$33 \mathrm{C} 66 \mathrm{~N}+\mathrm{A}$ & $6,8 \mathrm{C}$ & $2,0 \mathrm{C}$ & $30,0 \mathrm{E}$ & $2,9 \mathrm{D}$ \\
$100 \mathrm{~N}+\mathrm{A}$ & $2,8 \mathrm{E}$ & $4,8 \mathrm{~B}$ & $740,0 \mathrm{~B}$ & $104,5 \mathrm{~A}$ \\
\hline Controle & $8,4 \mathrm{~B}$ & 4,9 & 8,6 & 11,0 \\
\hline CV\% & 4,5 & &
\end{tabular}

${ }^{1}$ Médias na mesma coluna seguidas de diferentes letras são diferentes ( $\left.\mathrm{£} 0,05\right)$ pelo teste Tukey; ${ }^{2}$ 100C: 100\% de Crotalária Júncea; 66C33N: 66\% de Crotalária Júncea + 33\% de Napier; 33C66N: 33\% de Crotalária Júncea +66\% de Napier; 100N: 100\% de Napier; 33C66N+E: $33 \%$ de Crotalária Júncea + 66\% de Napier, inoculado com mais 5\% da massa com esterco bovino; 33C66N+A: 33\% de Crotalária Júncea + 66\% de Napier, inoculado com 100 litros de Agrobio diluído a 5\%; 100N+A: 100\% de Napier, inoculado com 100 litros de Agrobio diluído a $5 \%$ (mean values in the column, followed by the same letter did not differ from each other in the Tukey test (p£0,05); ${ }^{2} 100 \mathrm{C}: 100 \%$ Crotalária Júncea; 66C33N: 66\% Crotalária Júncea + 33\% Napier; 33C66N: 33\% Crotalária Júncea + 66\% Napier; 100N: 100\% Napier; 33C66N+E: $33 \%$ Crotalária Júncea $+66 \%$ Napier, innoculated with $5 \%$ of the mass of cattle manure; 33C66N+A: 33\% Crotalária Júncea + 66\% Napier, innoculated with 100L of 5\% diluted Agrobio; $100 \mathrm{~N}+\mathrm{A}$ : $100 \%$ Napier, inoculado com $100 \mathrm{~L}$ of 5\% diluted Agrobio). semeadura. Para as avaliações, foram utilizadas dez plantas por parcela. Foram avaliados a altura da parte aérea, número de folhas, produção de massa fresca na parte aérea e produção de massa seca na parte aérea.

\section{RESULTADOS E DISCUSSÃO}

Alface: Houve efeito significativo para todas as características estudadas (Tabela 2).

Os compostos produzidos apenas com Napier foram os que apresentaram os piores resultados, com desempenho muito inferior aos demais tratamentos. É provável que foi devido aos reduzidos teores de nutrientes (Tabela 1) e também devido à baixa estabilidade e maturidade destes compostos, que geralmente está associada à baixa capacidade de retenção de água, baixa CTC, $\mathrm{pH}$ muito elevado e presença de substâncias tóxicas.

O composto produzido apenas com Crotalária Júncea (100C) apresentou resultado inferior aos compostos produzidos com a mistura de Crotalária Júncea e Napier. A elevada salinidade apresentada por este composto, revelada por seu elevado valor de CE $\left(2,80 \mathrm{dS} \mathrm{m}^{-1}\right)$ pode ter ocasionado este resultado.

Os compostos produzidos com a mistura de Crotalária Júncea e Napier proporcionaram os melhores resultados, provavelmente devido apresentarem teores de nutrientes e outros sais em níveis ótimos para o desenvolvimento das mudas de alface.

Os tratamentos 66C33N, 33C66N+E e $33 \mathrm{C} 66 \mathrm{~N}+\mathrm{A}$ resultaram em valores de produção de massa seca de parte aérea muito próximos aos valores obtidos por Martins et al. (2001) para mudas de alface produzidas em substrato de vermicomposto. Frazin et al. (2005), obtiveram valores de 122 e 59 mg planta $^{-1}$ para produção de massa fresca e de massa seca, respectivamente, de mudas de alface Regina aos 20 dias após a semeadura e crescidas em substrato Plantmax sob condições controladas.

Os materiais com inoculação da mistura de 33\% de Crotalária Júncea e 66\% de Napier apresentaram desempenho semelhante ao tratamento 66C33N, in- 
dicando que se pode reduzir os níveis de Crotalária Júncea na mistura, desde que se efetue a inoculação (Tabela 2).

Beterraba: Houve efeito significativo para todas as características estudadas (Tabela 3).

Os compostos produzidos apenas com Napier também apresentaram os menores valores para as características estudadas.

O composto produzido com a mistura de 66\% de Crotalária Júncea e 33\% de Napier foi o que mais favoreceu o desenvolvimento das mudas de beterraba.

O composto produzido com a mistura de 33\% de Crotalária Júncea e 66\% de Napier, sem inoculação, apresentou desempenho semelhante ao substrato comercial. Os materiais com a inoculação desta mistura apresentaram desempenho inferior. O composto produzido apenas com Crotalária Júncea (100C) também apresentou desempenho inferior.

Os resultados obtidos para produção de massa fresca e de massa seca de parte aérea de mudas de beterraba estão, em média, superiores aos obtidos por Lopes et al. (2004) que, testando um substrato formado pela mistura de Plantmax e Argisol, obtiveram valores de 193,2 e 10,3 mg planta ${ }^{-1}$.

Tomate: Houve efeito significativo para todas as características estudadas (Tabela 4).

Os resultados das características altura das plantas, $n^{\circ}$ de folhas, produção de massa fresca e de massa seca observados para tomate, foram semelhantes aos obtidos para alface e beterraba. O composto produzido com a mistura de 66\% de Crotalária Júncea e 33\% de Napier foi o que se mostrou mais favorável ao desenvolvimento das mudas de tomate. Os demais compostos apresentaram resultados inferiores ao substrato comercial.

Os resultados observados nos três experimentos permitem concluir que compostos orgânicos produzidos com a mistura de 66\% de Crotalaria juncea L. e 33\% de capim Napier (Pennisetum purpureum Schum.) podem ser utilizados na produção de mudas de alface, beterraba e tomate.

\section{REFERÊNCIAS}

ABREU MF; ABREU CA; BATAGLIA OC. 2002. Uso da análise química na avaliação da qualidade de substratos e componentes. In: FURLANI AMC. Caracterização, manejo e qualidade de substratos para produção de plantas. Campinas: Instituto Agronômico, p.17-28. (IAC. Documentos 70).

BAUMGARTEN A. 2002. Methods of chemical and physical evaluation of substrate for plants. In: FURLANI AMC. Caracterização, manejo e qualidade de substratos para produção de plantas. Campinas: Instituto Agronômico, p.7-15. (IAC. Documentos 70)

CARLILE WR. 1997. The requirements of growing media. Peat in Horticulture 2: 17-23.

CORTI C; CRIPPA L. 1998. Compost use in plant nurseries: hydrological an physicochemical characteristics. Compost Science and Utilization 6: 35-45.

De BRITO AMA; GAGNE S. 1995. Effect of Compost on Rhizosphere Microflora of the Tomato and on the Incidence of Plant GrowthPromoting Rhizobacteria. Applied and Environmental Microbiology 61: 194-199.
FERMINO MH. 2002. O uso da análise física na avaliação da qualidade de componentes e substratos. In: FURLANI AMC. Caracterização, manejo e qualidade de substratos para produção de plantas. Campinas: Instituto Agronômico, p.29-37. (Documentos IAC, 70).

FRAZIN SM; MENEZES NL; GARCIA DC; SANTOS OS. 2005. Efeito da qualidade das sementes sobre a produção de mudas de alface. Horticultura Brasileira 23: 193-197.

HADAS A; PORTNOY R. 1997. Rates of decomposition in soil and release of available nitrogen from cattle manure and municipal solid waste. Compost Science and Utilization 5: 48-54.

LIEVENS B. 2001. Systemic resistance induced in cucumber against Pythium root rot by source separated household waste and yard trimmings composts. Compost Science and Utilization 9: 221-229.

LOPES JC; ZONIA JB; CAVATLE PC. 2004. Efeito de diferentes tratamentos e substratos na germinação e desenvolvimento de plântulas de beterraba. Horticultura Brasileira 22, suplemento CD-ROM.

MANDELBAUM R; HADAR Y. 1997. Methods for determining Pythium suppression in container media. Compost Science and Utilization 5: 15-22.

MARTINS ST; LUZ JQM; DINIZ KA. 2001. Produção de mudas de alface em substrato a base de vermicomposto. Horticultura Brasileira 19, suplemento CD-ROM.

SILVA FC. 1999. Manual de análises químicas de solos, plantas e fertilizantes. Brasília, DF: Embrapa Comunicação para Transferência de Tecnologia, $370 \mathrm{p}$.

TEDESCO MJ. 1995. Análise de solos, plantas e outros materiais. 2. ed. Porto Alegre: Departamento de Solos/UFRGS, 174 p.

WRAP - THE WASTES AND RESOURCES ACTION PROGRAMME. 2004. To support the development of standards for compost by investigating the benefits and efficacy of compost use in different applications. OxonUK, 72p. 